\section{Serum uric acid, kidney function and acute ischemic stroke outcomes in elderly patients: a single-cohort, perspective study}

Lorenzo Falsetti, 1 William Capeci,2,3 Nicola Tarquinio, 2,3 Giovanna Viticchi, 4 Mauro Silvestrini, 4 Vania Catozzo, $, 2,3$ Agnese Fioranelli, 2,3 Laura Buratti, 4 Francesco Pellegrini2,3

1Internal and Sub-intensive Medicine Department, Ospedali Riuniti Hospital, Ancona; 2Department of Internal Medicine, Santa Casa Hospital, Loreto (AN); 3Department of Internal Medicine, S.S. Benvenuto e Rocco Hospital, Osimo (AN); ${ }^{4}$ Neurological Clinic, Department of Experimental and Clinical Medicine, Marche Polytechnic University, Ancona, Italy

\begin{abstract}
Chronic kidney disease and hyperuricemia have been associated to an increased risk and a worse prognosis in acute ischemic stroke. Several mechanisms, including platelet dysfunction, coagulation disorders, endothelial dysfunction, inflammation, and an increased risk of atrial fibrillation could be implicated. The role of serum uric acid in this setting is still object of debate. We enrolled all the consecutive patients admitted to our department for acute ischemic stroke. Cox regression analysis was used to evaluate the risk of in-hospital death considering serum uric acid levels and all the comorbidities. In the overall sample, hyperuricemia was independently associated to an increased risk of in-hospital mortality. This effect was stronger in patients with chronic kidney disease while, in the group of patients with normal renal function, the relationship between hyperuricemia and increased stroke mortality was not confirmed. Hyperuricemia could be associated to higher in-hospital mortality for ischemic stroke among elderly patients when affected by kidney disease. Survival does not seem to be affected by hyperuricemia in patients with normal kidney function.
\end{abstract}

\section{Introduction}

Uric acid is the final product of purine metabolism. Different genetic mutations, which probably occurred later in the evolution, reduced the activity of uricase in higher primates and completely inactivated the gene activity in humans. Thus, serum uric acid (SUA) concentrations in humans are higher than in other primates with different uricase activity. Despite its intrinsic nature of waste product, $90 \%$ of the SUA filtered by the kidney is reabsorbed, suggesting that it could have played a role in the evolutionary process ${ }^{1}$ and could still exert some beneficial activities.

SUA is a major natural antioxidant and increased levels have been associated to a slower progression of several neurodegenerative disorders but also to an improvement in neurological and immune functions. ${ }^{1}$ In western world, normal cutoffs in adults have been set between 6.0 $\mathrm{mg} / \mathrm{dL}$ in females and $6.8 \mathrm{mg} / \mathrm{dL}$ in males. However, studies on isolated populations found that SUA levels range between 2.0 and $4.0 \mathrm{mg} / \mathrm{dL}$, suggesting that normal SUA concentrations could be lower. ${ }^{2}$ SUA levels may present significant changes in relation to age, sex, patients' physical characteristics, kidney function, diet, drugs and alcohol intake. Further, SUA levels increase in women after menopause, approaching those of men.

Human tissues are not able to metabolize uric acid ${ }^{1}$ and SUA homeostasis is maintained mainly by gut and kidney, which remove respectively $1 / 3$ and $2 / 3$ of the produced amount. SUA levels are a result of the balance between produced and excreted quantities: a decrease in kidney function significantly contributes to hyperuricemia. $^{3}$

Uric acid crystals adhere to the surface of epithelial cells so inducing an inflammatory response. Detrimental effects of hyperuricemia include increased serum levels of cytokines and tumor necrosis factor $\alpha$ and the local expression of chemokines, monocyte chemotactic protein and cyclooxygenase 2 in blood vessels. ${ }^{4}$ SUA levels seem also to be correlated to endothelial dysfunction, an early expression of vascular damage. 5

Several studies found an association between high SUA levels and hypertension, ${ }^{5}$ metabolic syndrome, ${ }^{6}$ chronic kidney disease (CKD), ${ }^{3}$ cardiovascular diseases (CVD), ${ }^{7}$ electrocardiographic alterations ${ }^{8}$ and non-valvular atrial fibrillation (NVAF). ${ }^{9}$ However, there is no clear evidence that treatment of hyperuricemia
Correspondence: Lorenzo Falsetti, Internal and Sub-intensive Medicine Department, Ospedali Riuniti Hospital, via Conca 10, 60126 Ancona, Italy.

Tel: +39.071.5965269/+39.338.7034188.

E-mail: drfalsetti@yahoo.it

Key words: Ischemic stroke; Serum uric acid; Chronic kidney failure; Aging.

Contributions: LF and GV, paper writing, study concept and design, data analysis and interpretation; WC and LB, study concept, data analysis and interpretation; NT, VC, and $\mathrm{AF}$, study concept, data acquisition and interpretation; MS and FP, study concept and design, study supervision, critical revision.

Conflict of interest: the authors declare no potential conflict of interest.

Received for publication: 6 October 2016.

Revision received: 29 January 2017.

Accepted for publication: 1 February 2017.

This work is licensed under a Creative Commons Attribution NonCommercial 4.0 License (CC BY-NC 4.0).

CCopyright L. Falsetti et al., 2017

Licensee PAGEPress, Italy

Neurology International 2017; 9:6920

doi:10.4081/ni.2017.6920

can significantly reduce cardiovascular risk.

The role of SUA in the acute phase of ischemic stroke (IS) has been investigated. 10 SUA concentrations increase in the first hours after IS and decrease to baseline levels in the following days. ${ }^{11}$ Beneficial anti-inflammatory effects of SUA have been described both in chronic. ${ }^{11}$ and acute pathologic conditions. ${ }^{12}$ Animal models seem to confirm a neuroprotective role for SUA in the setting of IS. In humans, an association between higher SUA levels and better outcomes after IS 12 has also been described. However, several mechanisms including platelet dysfunction, coagulation disorders, endothelial dysfunction, increased oxidative stress, thrombus formation, inflammation and increased risk of NVAF13 have been observed in hyperuricemic subjects, suggesting an association between SUA and worse prognosis after acute vascular diseases. To date, however, the exact pathogenic function of uric acid in acute cerebrovascular disorders is still not well established, and increased SUA levels could merely represent an alteration of purine metabolism or a reduction of SUA excretion in subjects with end-organ disease.

This single cohort, perspective study 
aims to evaluate the relationship between SUA levels and short-term outcomes of IS in a cohort of elderly patients.

\section{Materials and Methods}

\section{Study population}

In this single-cohort, perspective study, we enrolled all consecutive subjects admitted for IS to the Internal Medicine department of Santa Casa Hospital, Loreto (Italy), during a three-year period (2009-2011) within 24 hours after symptoms' onset and National Institutes of Health Stroke Scale (NIHSS) score $\geq 4$ and $\leq 25$. Patients with cerebral bleeding or tumours at brain CT scan, epileptic crisis at stroke onset and under treatment with xanthine oxidase inhibitors (allopurinol or febuxostat) were excluded. At the admission, each patient was submitted to a general, cardiological and neurological investigation. Patients undergoing systemic thrombolysis or requiring invasive ventilation in the emergency department (ED) were excluded from the analysis. All the subjects were diagnosed and treated according to international current guidelines. The local Ethics Committee approved the study protocol. Written, informed consent was obtained from each patient or from the caregiver. Investigations were made in accordance with the Declaration of Helsinki.

Age, sex, National Institutes of Health stroke scale (NIHSS), diabetes, hypertension, dyslipidaemia, heart failure (HF), CVD, NVAF, cancer, chronic obstructive lung disease (COPD), CKD, liver disease, peptic ulcer disease, presence of dementia and multimorbidity were evaluated and recorded at admission. Use of ACEInhibitors (ACE-I) or angiotensin receptor blockers (ARB), diuretics and antiplatelet agents was also investigated. A complete blood analysis, including serum creatinine and serum uric acid concentrations was obtained in the first 24 hours of admission in our Internal Medicine department. Days of hospitalization and in-hospital mortality were recorded. The primary outcome was defined as in-hospital mortality from any cause.

\section{Diabetes}

Diabetes was defined by a history of diabetes or the use of antidiabetic medications at the moment of the enrolment or by the presence of fasting plasma glucose $\geq 126$ $\mathrm{mg} / \mathrm{dL}$ or glycosylate haemoglobin $>6.5 \%$ during the hospitalization.

\section{Hypertension}

Hypertension was diagnosed in the presence of a previous history of increased blood pressure(BP) at home evaluation ( $\geq 135 \mathrm{mmHg}$ for systolic BP and $\geq 85$ $\mathrm{mmHg}$ for diastolic BP) reported by the patient or the caregiver or an increased BP ( $\geq 140 \mathrm{mmHg}$ for systolic BP and $\geq 90$ $\mathrm{mmHg}$ for diastolic BP) reported by the general practitioner. We considered adequate to confirm this comorbidity or a previous diagnosis of hypertension or antihypertensive drugs use at the moment of the enrolment. We did not use the BP levels found at the initial evaluation in the ED or during the hospitalization for stroke to put a new diagnosis of hypertension.

\section{Dyslipidaemia}

Dyslipidaemia was considered when the patient or the caregiver reported a positive history for this condition. Alternatively, we considered diagnostic the chronic use of a statin or ezetimibe to treat this specific condition or fasting LDL-C levels $>100 \mathrm{mg} / \mathrm{dL}$ during the hospitalization.

\section{Heart failure}

Heart failure was added to the list of comorbidities in the presence of at least one previous hospitalization for typical signs and symptoms and a subsequent clinical diagnosis of HF put by a cardiologist or an internal medicine specialist.

\section{Cardiovascular diseases}

Presence of CVD was underlined in presence of a history of previous myocardial infarction or angina.

\section{Non-valvular atrial fibrillation}

We considered a diagnosis of NVAF when the patient or the caregiver reported a history of this condition or when the patient reported a use of anticoagulant or antiarrhythmic agents for this pathology. We also diagnosed this condition if we observed it in the ECGs performed in the ED or during the hospitalization.

\section{Cancer}

A history of previous or active cancer was also investigated and added to the list of comorbidities in the database.

\section{Chronic obstructive lung disease}

COPD was considered when the patient or the caregiver reported this pathology at the first visit. We selected only patients with a diagnosis put by a lung specialist using spirometric data.

\section{Chronic kidney disease}

CKD was defined as the presence of a stable reduction in glomerular filtration rate (eGFR) eGFR $<60 \mathrm{ml} / \mathrm{min} / 1.73 \mathrm{~m}^{2}$ for a period $\geq 3$ months before hospital admission. We estimated eGFR according to chronic kidney disease epidemiology collaboration (CKD-EPI) equation ${ }^{14}$ in all subjects at the their arrival in the ED. Moderate-severe CKD was defined as eGFR $<60 \mathrm{~mL} / \mathrm{min} / 1.73 \mathrm{~m}^{2}$.

\section{Dementia}

Dementia was defined as the presence of a clinically relevant cognitive deterioration before the admission. We required a previous diagnosis put by a neurologist to confirm this condition. We enrolled subjects with both neurodegenerative and vascular forms of dementia.

\section{Multimorbidity}

We considered the presence of multimorbidity in presence of two or more chronic pathologies complicating IS in the same enrolled subject.

\section{Nosocomial infections}

From the admission in our department to the outcome (in-hospital death or end of hospitalization), we monitored and recorded the occurrence of hospital-acquired infections, defined as symptomatic urinary tract infections or pneumonia occurring at least 48 hours after hospital admission.

\section{Statistical analysis}

Age, NIHSS, days of hospitalization, SUA, serum creatinine and eGFR were recorded as continuous variables. Presence of diabetes, hypertension, dyslipidaemia, HF, CVD, NVAF, cancer, COPD, CKD, liver disease, peptic ulcer disease, dementia, presence of multimorbidity, hospitalacquired infections and in-hospital mortality were recorded as binary variables. SUA was also coded as a dichotomous variable with a cutoff at $7.0 \mathrm{mg} / \mathrm{dL}$, which was our laboratory standard cut-off. ACE-I or ARB, diuretics and antiplatelet drugs use was synthesized in different dichotomous variables.

Continuous variables were compared with the t-test, dichotomous and ordinal variables with $\chi^{2}$-test. Survival was evaluated first with Kaplan-Meier's curve. This model accounted of in-hospital death as the main outcome, days of admission as time variable and SUA, treated as dichotomous variable, as the main predictor.

We then assessed the hazard ratio (HR) with Cox proportional hazards model. We adjusted the model for the most common medical conditions observed in elderly 
patients associated to a worse IS outcome: sex, ${ }^{15}$ age, 15 NIHSS at the admission, ${ }^{15}$ hypertension, ${ }^{16}$ diabetes, ${ }^{15,17}$ HF, 18 NVAF, ${ }^{15,19}$ dyslipidaemia, ${ }^{19}$ cancer, ${ }^{20}$ COPD, ${ }^{21}$ multimorbidity, ${ }^{22}$ hospitalacquired infections and dementia. 23

The multivariate model adopted in-hospital death as the main outcome, days of hospitalization as the time variable and SUA, treated as dichotomous variable, as the main predictor. The same analysis was run in the whole sample, in the CKD subgroup and in the group of patients with normal kidney function. We then performed a receiver operating characteristic (ROC) curve analysis to assess the optimal cut-off value for SUA in predicting in-hospital mortality in the subgroup of patients with kidney disease and prepared a second binary variable containing the newly obtained cut-off value. Last, we have run a Cox proportional hazards model adopting in-hospital death as the main outcome, days of hospitalization as time variable and SUA, treated as dichotomous variable with the new cutoff, as the main predictor. We adjusted this model with the same covariates considered in the first Cox regression: sex, age, NIHSS at the admission, hypertension, diabetes, HF, NVAF, dyslipidaemia, cancer, COPD, multimorbidity, hospital-acquired infections and dementia. The same model was tested in the group of subgroup of CKD patients and in the subgroup of subjects with preserved renal function. Statistical analysis was performed with SPSS 13.0 for Windows systems.

\section{Results}

332 subjects were evaluated for enrolment during the study interval. Of these, 16 required invasive ventilation in the $\mathrm{ED}, 18$ were sent to the hub hospital for thrombolysis and 4 refused to participate in the study. 18 subjects were not enrolled for treatment with xanthine-oxidase inhibitors at their arrival in the ED, and 4 subjects were excluded for incomplete data collection. We obtained a final sample of 272 patients. Baseline characteristics of the sample, inhospital mortality, duration of hospital admission and differences in the subpopulations according SUA levels are synthesized in Table 1.

Among subjects with CKD, we observed a mean SUA of $6.42( \pm 1.83)$ $\mathrm{mg} / \mathrm{dL}$ among survivors and a mean SUA of $7.61( \pm 1.92) \mathrm{mg} / \mathrm{dL}$ among patients who died during the hospitalization. Among patients with a normal renal function, we observed a mean SUA of $4.95( \pm 1.43)$ $\mathrm{mg} / \mathrm{dL}$ among survivors and a mean SUA of $5.00( \pm 1.90) \mathrm{mg} / \mathrm{dL}$ among patients who died during hospitalization.

Subdividing the sample according the presence of $\mathrm{CKD}$, defined as $\mathrm{eVFG}<60$ $\mathrm{mL} / \mathrm{m} 2$, we observed, in the population affected by CKD, a higher age (CKD:

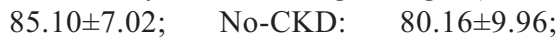
$\mathrm{P}<0.0001$ ), higher prevalence of HF (CKD: 46.2\%; No-CKD: $29.1 \%$; $\mathrm{P}=0.004)$, NVAF (CKD: $26.9 \%$, No-CKD: $10.9 \%$; $\mathrm{P}=0.001$ ) and multimorbidity (CKD: $70.6 \%$; NoCKD: $56.4 \% ; \mathrm{P}=0.018)$. Subjects with
CKD did not significantly differ from subjects with normal renal function in the use of ACE-I or ARB (CKD: 32.2\%; No-CKD: $22.0 \% ; \mathrm{P}=0.074$ ), diuretics (CKD: $77.7 \%$; No-CKD: $70.0 \% ; \mathrm{P}=0.172$ ) and antiplatelet agents (CKD: 15.2\%; No-CKD: $12.5 \%$; $\mathrm{P}=0.602)$.

Patients with SUA $\geq 7.0 \mathrm{mg} / \mathrm{dL}$ had an increased risk of in-hospital mortality at Kaplan-Meier's regression $(\mathrm{P}<0.05, \log$ rank test). The first Cox regression model, corrected for sex, age, NIHSS at the admission, hypertension, diabetes, HF, NVAF, dyslipidaemia, cancer, COPD, multimorbidity, in-hospital infections and dementia showed that, in the overall sample, SUA levels $\geq 7.0 \mathrm{mg} / \mathrm{dL}$ were independently associated to an increased risk of in-hospital mortality (HR:2.857; 95\%CI:1.022-7.983; $\mathrm{P}=0.045$ ), as shown in Figure $1 \mathrm{~A}$ and Table 2. This effect was more evident among patients with moderate-severe CKD (HR:7.977; 95\%CI:1.344-47.346; $\mathrm{P}=0.022)$, as shown in Figure $1 \mathrm{~B}$ and Table 3. Among patients with normal kidney function SUA was not significantly associated to an increased risk of in-hospital mortality (HR:3.632; $\quad 95 \% \mathrm{CI}: 0.277-47.633$; $\mathrm{P}=0.326$ ), as shown in Figure $1 \mathrm{C}$ and Table 4. ROC curve analysis, performed in the subgroup of patients with CKD, showed that SUA had a moderate but significant value in predicting in-hospital death (AUC: 0.683 ; $95 \% \mathrm{CI}: 0.503-0.862 ; \mathrm{P}=0.047$ ), as shown in Figure 2A. The best SUA cut-off value derived from this analysis in this subgroup was $\geq 7.35 \mathrm{mg} / \mathrm{dL} \quad(\mathrm{Se}: 72.7 \%$;

Table 1. Baseline characteristics of the sample according serum uric acid levels (cut-off $\geq 7.0 \mathrm{mg} / \mathrm{dL}$ ).

\begin{tabular}{|c|c|c|c|c|}
\hline Variable & Overall $(\mathrm{n}=272)$ & SUA $<7.0 \mathrm{mg} / \mathrm{dL} \quad(\mathrm{n}=220)$ & $\mathrm{SUA} \geq 7.0 \mathrm{mg} / \mathrm{dL} \quad(\mathrm{n}=52)$ & $\mathbf{P}$ \\
\hline Age $( \pm \mathrm{SD})$ (years)* & $82.37( \pm 9.19)$ & $81.29( \pm 9.62)$ & $86.00( \pm 5.89)$ & 0.001 \\
\hline Sex (\% of females) & $149(54.7)$ & $120(54.5)$ & $29(55.8)$ & 1.000 \\
\hline Days of Admission $( \pm \mathrm{SD})$ (days) & $9.87( \pm 7.14)$ & $10.23( \pm 8.25)$ & $9.84( \pm 4.91)$ & 0.747 \\
\hline Creatinine Clearance $( \pm \mathrm{SD})(\mathrm{mL} / \mathrm{min})^{*}$ & $64.65( \pm 26.06)$ & $69.63( \pm 24.41)$ & $46.47( \pm 23.84)$ & 0.0001 \\
\hline NIHSS $( \pm$ SD) & $7.74( \pm 0.89)$ & $7.75(0.91)$ & $7.67(0.90)$ & 0.556 \\
\hline In-hospital death $(\mathrm{n}, \%)^{*}$ & $19(7.70)$ & $10(3.7)$ & $9(17.3)$ & 0.003 \\
\hline Hypertension (n, \%) & $149(54.1)$ & $117(53.2)$ & $32(61.5)$ & 0.353 \\
\hline Diabetes (n, \%) & $52(18.4)$ & $47(21.4)$ & $5(9.6)$ & 0.076 \\
\hline Heart failure $(n, \%)^{*}$ & $96(36.4)$ & $68(30.9)$ & $28(53.8)$ & 0.002 \\
\hline Atrial fibrillation (n, \%) & $46(16.9)$ & $34(15.5)$ & $12(23.1)$ & 0.217 \\
\hline Dyslipidaemia (n, \%) & $33(12.1)$ & $30(13.6)$ & $3(5.8)$ & 0.157 \\
\hline Cancer (n, \%) & $11(4.04)$ & $8(3.6)$ & $3(5.8)$ & 0.445 \\
\hline COPD (n, \%) & $28(10.3)$ & $20(9.1)$ & $8(15.4)$ & 0.204 \\
\hline CKD $(\mathrm{n}, \%)^{*}$ & $112(41.2)$ & $72(32.7)$ & $40(76.9)$ & 0.000 \\
\hline Dementia $(\mathrm{n}, \%) *$ & $37(13.6)$ & $35(15.9)$ & $2(3.8)$ & 0.023 \\
\hline Hospital-acquired infections (n, \%) & $25(9.19)$ & $18(8.2)$ & $7(13.5)$ & 0.283 \\
\hline Multimorbidity (\%) ( $\geq 2$ chronic pathologies) & $170(62.5)$ & $132(60)$ & $38(73.1)$ & 0.083 \\
\hline
\end{tabular}

SUA, serum uric acid; SD, standard deviation; NIHSS, National Institutes of Health Stroke Scale; COPD, chronic obstructive pulmonary disease; CKD, chronic kidney disease. * Significant differences are marked with an asterisk. 
Sp:72.3\%; LR+:2.62; 95\%CI:1.6-4.2; LR$: 0.38 ; 95 \%$ CI:0.1-1.0). By applying this optimized cut-off to the Cox model, we confirmed, among patients affected by CKD with SUA $\geq 7.35 \mathrm{mg} / \mathrm{dL}$, an increased risk of in-hospital death for IS (HR:8.734; 95\%CI:1.514-50.379; $\mathrm{P}=0.015)$. The same cut-off confirmed an increased risk when applied to the general population (HR:3.518; 95\%CI: 1.284-9.636; $\mathrm{P}=0.014$ ), while, in the subset of patients with normal renal function, SUA did not show any predictive value (HR:13.889; 95\%CI:0.710$271.881 ; \mathrm{P}=0.083$ ) even with this new cutoff. Among patients with normal renal function we observed that ROC curve analysis of SUA was not significantly associated to in-hospital mortality (AUC:0.470; 95\%CI:0.245-0.694; $\mathrm{P}=0.772$ ), as shown in Figure 2B.

\section{Discussion}

Stroke is among the three most common cause of death, as well as the fourth leading cause of productivity loss and a major cause of disability worldwide. The epidemiological impact increases with aging. The identification and correction of clinically relevant risk factors is the most relevant approach in the attempt to reduce its incidence and improve patients' outcome.

Hyperuricemic patients have a greater risk of in-hospital mortality for IS independently from the presence of several common comorbidities and a relationship between stroke severity and SUA levels has already been hypothesized. 10

Hyperuricemic patients, however, have an increased prevalence of specific condi-
Table 2. Multivariate Cox regression analysis in the overall sample.

\begin{tabular}{lcccc} 
Variable & P & HR & Lower 95\%CI & Upper 95\% CI \\
Sex & 0.778 & 0.863 & 0.31 & 2.401 \\
Age & 0.039 & 1.087 & 1.004 & 1.176 \\
\hline Hypertension & 0.624 & 0.78 & 0.289 & 2.105 \\
Diabetes & 0.253 & 0.389 & 0.077 & 1.961 \\
\hline HF & 0.534 & 1.401 & 0.484 & 4.059 \\
NVAF & 0.187 & 0.397 & 0.1 & 1.568 \\
\hline Dyslipidemia & 0.981 & -- & -- & - \\
Cancer & 0.987 & -- & -- & -- \\
\hline COPD & 0.604 & 0.565 & 0.065 & 4.875 \\
Dementia & 0.288 & 0.316 & 0.038 & 2.645 \\
\hline Multimorbidity & 0.49 & 1.219 & 0.695 & 2.138 \\
Hospital-acquired Infections & 0.422 & 1.727 & 0.455 & 6.549 \\
\hline NIHSS & 0.169 & 1.443 & 0.856 & 2.432 \\
SUA $\left(^{*}\right.$ ) (cut-off $\left.>=7.0 \mathrm{mg} / \mathrm{dL}\right)$ & 0.045 & 2.857 & 1.022 & 7.983
\end{tabular}

$\mathrm{HR}$, hazard ratio; CI, confidence interval; $\mathrm{HF}$, heart failure; NVAF, non-valvular atrial fibrillation; COPD, chronic obstructive pulmonary disease; NIHSS, National Institutes of Health Stroke Scale; SUA, serum uric acid. *Significant variables are marked with an asterisk.

Table 3. Multivariate Cox regression analysis in the subpopulation with moderate-severe chronic kidney disease.

\begin{tabular}{lcccc} 
Variable & P & HR & Lower 95\%CI & Upper 95\%Cl \\
Sex & 0.92 & 1.073 & 0.273 & 4.212 \\
Age & 0.624 & 1.03 & 0.914 & 1.161 \\
\hline Hypertension & 0.551 & 0.651 & 0.158 & 2.674 \\
Diabetes & 0.611 & 1.6 & 0.261 & 9.808 \\
\hline HF & 0.754 & 1.261 & 0.295 & 5.386 \\
NVAF & 0.687 & 0.695 & 0.119 & 4.072 \\
\hline Dyslipidemia & 0.988 & - & - & - \\
Cancer & 0.993 & - & - & - \\
\hline COPD & 0.881 & 1.202 & 0.107 & 13.518 \\
Dementia & 0.607 & 1.928 & 0.158 & 23.581 \\
\hline Multimorbidity & 0.127 & 1.928 & 0.829 & 4.484 \\
Hospital-acquired Infections & 0.967 & 0.954 & 0.099 & 9.215 \\
\hline NIHSS & 0.808 & 0.913 & 0.437 & 1.906 \\
SUA (cut-off $>=7.0 \mathrm{mg} / \mathrm{dL})$ & 0.022 & 7.977 & 1.344 & 47.346 \\
\hline
\end{tabular}

$\mathrm{HR}$, hazard ratio; CI, confidence interval; $\mathrm{HF}$, heart failure; NVAF, non-valvular atrial fibrillation; COPD, chronic obstructive pulmonary disease; NIHSS, National Institutes of Health Stroke Scale; SUA, serum uric acid. *Significant variables are marked with an asterisk.
A

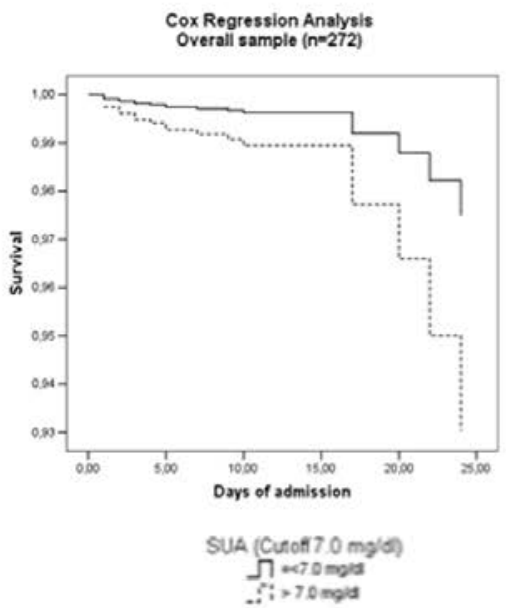

B

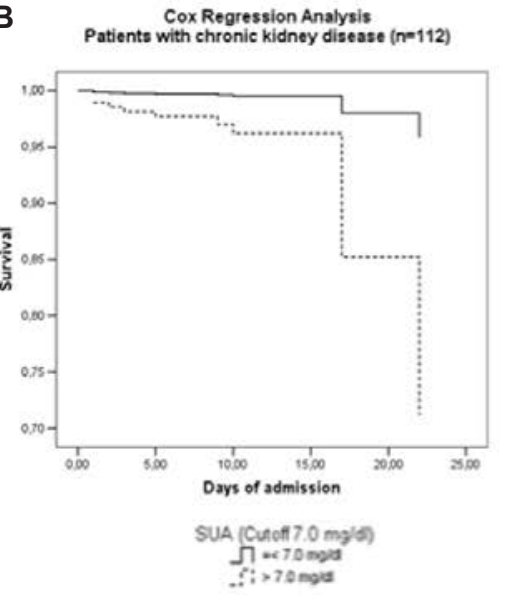

C Cox Regression Analysis.

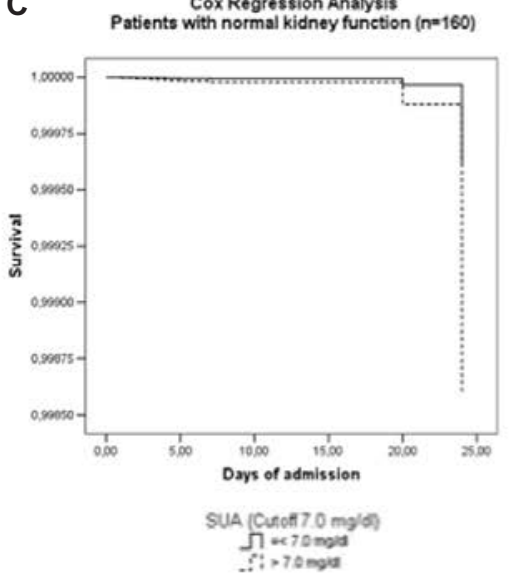

Figure 1. Cox proportional hazards model survival function in the complete sample (A), in the subgroup of patients (B) affected by chronic kidney disease, and with normal renal function $(C)$, adopting a serum uric acid cut-off $\leq 7.0 \mathrm{mg} / \mathrm{dL}$. 
tions, such as older age, ${ }^{24}$ poorly controlled hypertension, ${ }^{8} \mathrm{HF},{ }^{18} \mathrm{CKD},{ }^{25}$ metabolic syndrome, ${ }^{6} \quad \mathrm{CVD}^{7}{ }^{7}$ NVAF, ${ }^{9}$ and multimorbidity, 22 which are potentially involved in increasing stroke risk and negatively influencing stroke outcome. For this reason, the role of SUA as an independent risk factor for an unfavourable outcome in stroke patients is still widely controversial.

In the present study, hyperuricemia resulted independently associated to the main outcome in the overall sample and in the subgroup of patients affected by CKD, while this effect was not significant in patients with normal kidney function. This confirms a previous observation in which hyperuricemia was not associated with stroke outcomes in populations with low CKD prevalence. ${ }^{26}$ Increased SUA levels are associated to a decline in renal function and a poorly controlled blood pressure in hypertensive patients, which could partially explain the relationship with the clinical outcome. ${ }^{8}$ In fact, a poorly controlled hypertension is a recognized risk factor for worse functional outcomes and increased risk of death in IS, 27 and CKD, a recognized high-risk condition for vascular diseases, is a strong independent predictor of mortality and poor outcome in IS. ${ }^{28}$

According to our results, we can speculate that SUA could gather a clinically significant role in the prognosis of IS in specific, high-risk situations, such as CKD, probably by reducing blood pressure control, which is associated to an increased oedema in the acute phase of IS. ${ }^{27}$ Increased SUA levels have recently been associated to aspirin resistance, 29 which could directly affect IS outcomes: this effect could be stronger among patients affected by CKD, who usually show higher SUA levels and a higher vascular risk than the general population. It is not possible, however, to exclude that hyperuricemia could also act as a mere bystander of other stronger risk factors associated to a worse prognosis in IS, such as NVAF or HF. Current literature does not support the treatment of asymptomatic hyperuricemia even among subjects at high cardiovascular risk, 30 and the usefulness of reducing SUA with xanthine oxidase inhibitors in the setting of IS and other acute illnesses still requires further evaluation with appropriately designed randomized controlled trials.

The present study has several limitations. First, SUA levels may present significant fluctuations, making it difficult to obtain appropriate determinations. In this respect, we did not perform any additional measurement of SUA after the admission, and the study misses information about SUA levels changes during the follow-up

A

Patients affected by chronic kidney disease
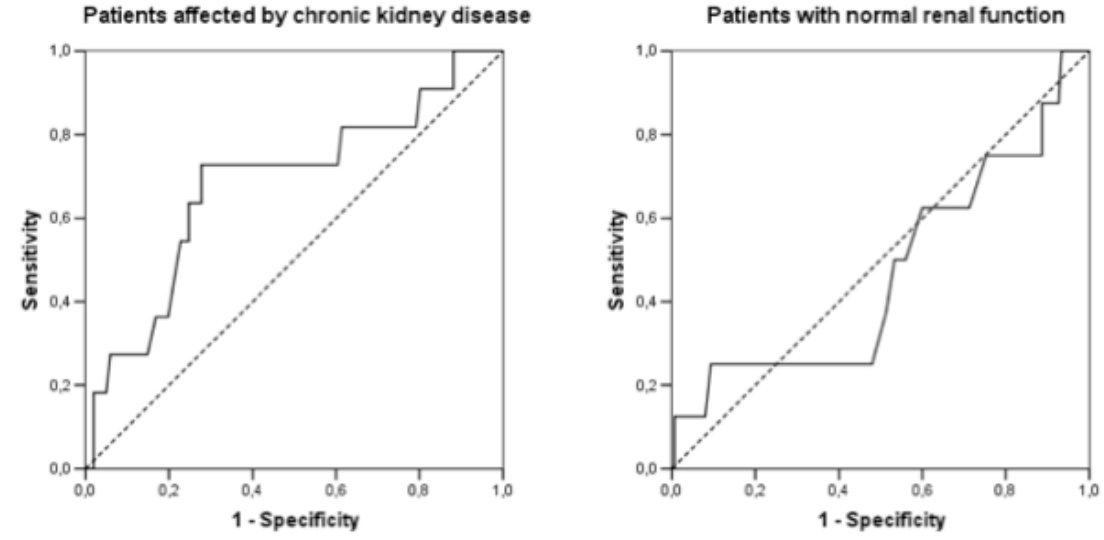

Figure 2. Receiver operating characteristic curve analysis for in-hospital death predicted by serum uric acid levels in patients affected by chronic kidney disease (A) and in patients with normal renal function (B).

Table 4. Multivariate Cox regression analysis in the subpopulation with normal renal function.

\begin{tabular}{lcccc} 
Variable & P & HR & Lower 95\%CI & Upper 95\%CI \\
Sex & 0.696 & 0.646 & 0.072 & 5.793 \\
\hline Age & 0.057 & 1.119 & 0.996 & 1.256 \\
Hypertension & 0.547 & 0.555 & 0.082 & 3.773 \\
\hline Diabetes & 0.957 & - & - & - \\
HF & 0.535 & 1.945 & 0.237 & 15.942 \\
\hline NVAF & 0.426 & 0.36 & 0.029 & 4.445 \\
Dyslipidemia & 0.985 & - & - & - \\
\hline Cancer & 0.993 & - & - & - \\
COPD & 0.985 & - & - & - \\
\hline Dementia & 0.98 & - & - & - \\
Multimorbidity & 0.8 & 0.888 & 0.354 & 2.229 \\
\hline Hospital-acquired Infections & 0.026 & 18.105 & 1.416 & 14.043 \\
NIHSS & 0.022 & 4.157 & 1.23 & 47.633 \\
\hline SUA (cut-off $>=7.0 \mathrm{mg} / \mathrm{dL})$ & 0.326 & 3.632 & 0.277 &
\end{tabular}

$\mathrm{HR}$, hazard ratio; $\mathrm{Cl}$, confidence interval; $\mathrm{HF}$, heart failure; NVAF, non-valvular atrial fibrillation; COPD, chronic obstructive pulmonary disease; NIHSS, National Institutes of Health Stroke Scale; SUA, serum uric acid. *Significant variables are marked with an asterisk.

period. Several commonly used drugs significantly affect SUA concentrations, and this could represent an important limit of our observations. In fact, we observed similar proportions in ACE-I, ARB, antiplatelet and diuretics use in patients with and without CKD, but we were not able to correct our models for the dose taken, especially for diuretics.

\section{Conclusions}

In conclusion, our study can only be considered as preliminary and able to suggest hypotheses. However, in relation to the possible practical implications of the demonstration of a significant role of SUA in the clinical evolution of stroke patients, it would be useful the planning of more extensive investigations to confirm our findings.

\section{References}

1. Álvarez-Lario B, Macarrón-Vicente J. Uric acid and evolution. Rheumatology 2010;49:2010-5.

2. Johnson RJ, Andrews P. Fructose, uricase, and the Back-to-Africa hypothesis. Evol Anthropol News Rev 2010;19: 250-7.

3. Prasad Sah OS, Qing YX. Associations between hyperuricemia and chronic kidney disease: a review. Nephrourol Mon 2015;7:3. 
4. Kang D-H, Park S-K, Lee I-K, Johnson RJ. Uric acid-induced C-reactive protein expression: implication on cell proliferation and nitric oxide production of human vascular cells. J Am Soc Nephrol 2005;16:3553-62.

5. Gagliardi ACM, Miname MH, Santos RD. Uric acid: a marker of increased cardiovascular risk. Atherosclerosis 2009;202:11-7.

6. Fu S, Luo L, Ye P, Xiao W. Epidemiological associations between hyperuricemia and cardiometabolic risk factors: a comprehensive study from Chinese community. BMC Cardiovasc Disord 2015;15:129.

7. Soltani Z, Rasheed K, Kapusta DR, Reisin E. Potential role of uric acid in metabolic syndrome, hypertension, kidney injury, and cardiovascular diseases: is it time for reappraisal? Curr Hypertens Rep 2013;15:175-81.

8. Cicero AFG, Rosticci M, Tocci G, et al. Serum uric acid and other short-term predictors of electrocardiographic alterations in the Brisighella Heart Study cohort. Eur J Intern Med 2015;2015:37.

9. Tamariz L, Agarwal S, Soliman EZ, et al. Association of serum uric acid with incident atrial fibrillation (from the Atherosclerosis Risk in Communities [ARIC] Study). Am J Cardiol 2011;108: 1272-6.

10. Chiquete E, Ruiz-Sandoval JL, MurilloBonilla LM, et al. Serum uric acid and outcome after acute ischemic stroke: PREMIER study. Cerebrovasc Dis 2013;35:168-74.

11. Waring WS. Uric acid: an important antioxidant in acute ischaemic stroke. QJM 2002;95:691-3.

12. Chamorro Á, Obach V, Cervera Á, et al. Prognostic significance of uric acid serum concentration in patients with acute ischemic stroke. Stroke
2002;33:1048-52.

13. Falsetti L, Viticchi G, Tarquinio N, et al. CHA2DS2-VASc in the prediction of early atrial fibrillation relapses after electrical or pharmacological cardioversion. J Cardiovasc Med 2014;15:63641.

14. Stevens LA, Schmid CH, Greene T, et al. Comparative performance of the CKD Epidemiology collaboration (CKD-EPI) and the modification of diet in renal disease (MDRD) Study equations for estimating GFR levels above $60 \mathrm{~mL} / \mathrm{min} / 1.73 \mathrm{~m} 2$. Am J Kidney Dis 2010;56:486-95.

15. Heuschmann PU, Kolominsky-Rabas PL, Misselwitz B, et al. Predictors of inhospital mortality and attributable risks of death after ischemic stroke: the German Stroke Registers Study Group. Arch Intern Med 2004;164:1761-8.

16. Lewington S, Clarke R, Qizilbash N, et al. Age-specific relevance of usual blood pressure to vascular mortality: a meta-analysis of individual data for one million adults in 61 prospective studies. Lancet 2002;360:1903-13.

17. Hamidon BB, Raymond AA. The impact of diabetes mellitus on in-hospital stroke mortality. J Postgrad Med 2017;49:307-10.

18. Haeusler KG, Laufs U, Endres M. Chronic heart failure and ischemic stroke. Stroke 2011;42:2977-82.

19. Smith EE, Shobha N, Dai D, et al. A risk score for in-hospital death in patients admitted with ischemic or hemorrhagic stroke. J Am Heart Assoc 2013;2:1.

20. Cutting S, Wettengel M, Conners JJ, et al. Three-month outcomes are poor in stroke patients with cancer despite acute stroke treatment. J Stroke Cerebrovasc Dis 2016;24:1052-3057(16)30423-2.

21. Lekoubou A, Ovbiagele B. Prevalence and influence of chronic obstructive pulmonary disease on stroke outcomes in hospitalized stroke patients. eNeurologicalSci 2017;6:21-4.

22. Falsetti L, Viticchi G, Tarquinio N, et al. Charlson comorbidity index as a predictor of in-hospital death in acute ischemic stroke among very old patients: a single-cohort perspective study. Neurol Sci 2016;37:1443-8.

23. Barba R, Morin M-M, Cemillán C, et al. Previous and incident dementia as risk factors for mortality in stroke patients. Stroke 2002;33:1993-8.

24. Nakayama H, Jørgensen HS, Raaschou $\mathrm{HO}$, Olsen TS. The influence of age on stroke outcome. The Copenhagen Stroke Study. Stroke 1994;25:808-13.

25. Wang Z, Lin Y, Liu Y, et al. Serum uric acid levels and outcomes after acute ischemic stroke. Mol Neurobiol 2015; 53:1753-9.

26. Miedema I, Uyttenboogaart M, Koch $\mathrm{M}$, et al. Lack of association between serum uric acid levels and outcome in acute ischemic stroke. J Neurol Sci 2012;319:51-5.

27. Leonardi-Bee J, Bath PMW, Phillips SJ, Sandercock PAG. Blood pressure and clinical outcomes in the International Stroke Trial. Stroke 2002;33:5.

28. Yahalom G, Schwartz R, Schwammenthal Y, et al. Chronic kidney disease and clinical outcome in patients with acute stroke. Stroke 2009;40:4.

29. Yildiz BS, Ozkan E, Esin F, et al. Does high serum uric acid level cause aspirin resistance? Blood Coagul Fibrinolysis 2016;27:412-8.

30. Pasina L, Brucato AL, Djade CD, et al. Inappropriate prescription of allopurinol and febuxostat and risk of adverse events in the elderly: results from the REPOSI registry. Eur J Clin Pharmacol 2014;70:1495-503. 